\title{
Repercusión de la formación investigadora en la producción científica de Expresión Gráfica Arquitectónica indexada en SJR
}

\author{
Vicente López-Chao*, Antonio Amado Lorenzo*
}

*Departamento de Expresión Gráfica Arquitectónica, Escuela Superior de Arquitectura de A Coruña,Universidade da Coruña Correo-e: v.lchao@udc.es | ORCID iD: https://orcid.org/0000-0002-7369-0319

Correo-e: antonio.amado@udc.es | ORCID iD: http://orcid.org/0000-0002-4208-4463

Recibido: 03-07-20; 2a versión: 05-10-20; Aceptado: 05-10-20; Publicado: 20-10-21

Cómo citar este artículo/Citation: López-Chao, V.; Amado Lorenzo, A. (2021). Repercusión de la formación investigadora en la producción científica de Expresión Gráfica Arquitectónica indexada en SJR. Revista Española de Documentación Científica, 44 (4), e306. https://doi.org/ 10.3989/redc.2021.4.1814

Resumen: Los sistemas de evaluación del rol investigador del profesorado universitario español se centran en el artículo científico como unidad de medición de los avances de un área de conocimiento. Se ha constatado que los estudios globales pueden proporcionar resultados sesgados y contradictorios. En España, la arquitectura se evalúa junto a las ingenierías sin considerar sus vertientes técnicas o artísticas específicas. Este estudio se centra en conocer la producción científica en SJR del profesorado del área Expresión Gráfica Arquitectónica de todas las escuelas de arquitectura públicas españolas $(n=292)$, y si ésta se relaciona con el grado de doctor y la figura académica. Para ello se utilizará estadística descriptiva, comparativa e inferencial. Los resultados revelan índices de publicación bajos y una tendencia de crecimiento en la última década, pudiéndose relacionar los mejores resultados con la formación de doctor y figura contractual.

Palabras clave: arquitectura; expresión gráfica arquitectónica; educación superior; bibliometría; investigación científica.

\section{Research training impact on the SJR scientific production of Architectural Graphics}

Abstract: The research role evaluation systems of the Spanish university teaching staff focus on the scientific paper as a unit for measuring progress in an area of knowledge. Studies covering many areas may provide biased and conflicting results. Architecture is evaluated together with engineering without considering their technical or artistic aspects. This study seeks to identify the SJR scientific production of the Architectural Graphics area teaching staff of all the Spanish public architecture schools $(n=292)$, and if it is related to the degree of doctor and the academic figure. For this, descriptive, comparative and inferential statistics are used. The results have revealed low publication rates and an increasing trend in the last decade, as well as a direct relationship between better publication rates with research training and the academic rank.

Keywords: architecture; architectural graphics; higher education; bibliometrics; scientific research.

Copyright: (c) 2021 CSIC. Este es un artículo de acceso abierto distribuido bajo los términos de la licencia de uso y distribución Creative Commons Reconocimiento 4.0 Internacional (CC BY 4.0). 


\section{INTRODUCCIÓN}

Entre otras cosas, la evolución del conocimiento se relaciona con los avances en investigación. En las últimas décadas se ha desarrollado un consenso sobre la evaluación de la calidad investigadora en función de parámetros como la producción científi$\mathrm{ca}$, entendiendo las publicaciones científicas como el principal índice de calidad del conocimiento. Por ello, los estudios de indicadores bibliométricos han ganado importancia en los últimos años.

Sin embargo, no todas las publicaciones de este tipo tienen el mismo nivel de reconocimiento, calidad o visibilidad (Osca-Lluch y otros, 2019). Uno de los indicadores más fiables es la indexación de la revista en listados periódicos como los de Journal Citation Reports (JCR) o Scimago Journal Citation Rank (SJR).

El caso concreto de la arquitectura es preocupante ya que en muchos ámbitos su evaluación se vincula a áreas de ingeniería, por lo que los datos aportados no son totalmente representativos. De hecho, la indexación JCR mencionada anteriormente, la mejor valorada en méritos de promoción académica, ni siquiera contempla la arquitectura como un área específica, lo que supone una contradicción con los mencionados rankings globales. Si bien es verdad que existen categorías como Construction and Building Technology o Civil Engineering, ambas tienen que ver con áreas técnicas de la arquitectura como estructuras, instalaciones o construcción, las cuales, a nivel internacional, se atribuyen tradicionalmente al campo de la ingeniería. Mientras que el diseño y el proyecto, ejes de la formación en arquitectura, no se verían representados. De hecho, las revistas que responden a estos alcances se indexan normalmente en la categoría de Visual Arts and Performing Arts de SJR. Esta situación se acentúa todavía más en sub-áreas particulares como la Expresión Gráfica Arquitectónica (EGA) con una gran implicación del componente artístico. En este marco, las revistas reconocidas por la CNEAI y las más utilizadas por la comunidad equivalen a aquellas indexadas en SCOPUS/SJR por lo que son un marco adecuado para el estudio.

Este escenario permite evidenciar la producción en áreas que "funcionan bien" en términos de producción científica, pero impide conocer la realidad investigadora exigida por las instituciones evaluadoras en áreas y sub-áreas más peculiares y específicas. En este sentido esta investigación puede servir como aportación para que los procesos de evaluación de investigadores de este campo del conocimiento sean más objetivos. Para ello, se busca diagnosticar los niveles de producción científica en un área artístico-técnica que requiere de una fuerte componente profesional. Y posteriormente, se pretende averiguar si la producción científica en EGA se relaciona con la formación investigadora teniendo en cuenta el grado de doctor y la figura académica, con el objetivo de que sirva como eje vertebrador del cambio hacia su profesionalización científica. Por lo que ayudará a clarificar la rentabilidad científica de realizar una tesis doctoral en arquitectura.

\section{ANTECEDENTES Y OBJETIVOS}

\subsection{Relevancia de EGA en investigación}

La expresión gráfica es el lenguaje vehicular habitual de los arquitectos que permite comunicar información compleja muy difícil de transmitir únicamente con palabras. De hecho, la investigación en EGA utiliza fuentes de información muy particulares, como dibujos históricos y técnicas de levantamiento de planos sobre el patrimonio construido (Chías y Abad, 2018), muestras de fachadas de edificios clásicos para determinar su estado cromático original (Llopis y otros, 2015), o fotografías y dibujo asistido por ordenador para realizar análisis comparativos (Gámiz-Gordo y Ferrer Pérez Blanco, 2019), entre muchos otros campos de investigación.

Este tipo de investigación ha desarrollado diversas teorías sobre técnicas de comunicación propias de este lenguaje como el dibujo en arquitectura y el nuevo paradigma de la era digital (Llopis Verdú, 2018a; Llopis Verdú, 2018b), la confrontación entre la representación hiper-realista entre la fotografía y la infografía (Franco Taboada, 2011), las posibilidades del dibujo digital a mano alzada (Amado Lorenzo y Fraga López, 2015) o la maqueta como herramienta de representación de la ciudad y la arquitectura (Carazo Lefort, 2018; Ortega y otros, 2012). Además, el área se ha centrado en fundamentos del dibujo arquitectónico como la línea, el color y la perspectiva (Ampliato Briones, 2012; Franco Taboada, 2015; Marcos Alba y otros, 2017).

Otros avances se han relacionado con la visualización arquitectónica como la representación digital a través de realidad aumentada (Fonseca y otros, 2014; Redondo y otros, 2012) o la digitalización de edificios a través de técnicas fotogramétricas o de escáner láser (Soler-Estrela, 2018; Alonso Rodríguez y Aliberti, 2019; Ferrer-Pérez-Blanco y otros, 2019; Reinoso-Gordo y otros, 2018). Así como el desarrollo de protocolos para la gestión de la documentación gráfica de edificios patrimoniales a través de técnicas Building Information Modelling (Jordan-Palomar y otros, 2018). 
Este conjunto de fuentes de información y técnicas de comunicación y visualización trascienden en una investigación artístico-científica muy específica que permite desarrollar el análisis descriptivo, constructivo o espacial de entornos construidos (Parra Bañón, 2008; Pinto, 2013; Natapov y otros, 2020) y que ahondan en el significado y función del patrimonio construido (Franco Taboada y Goy Diz, 2018).

Por todo ello, es necesario diagnosticar el estado de la producción científica de calidad en EGA que nada tiene que ver con otras áreas de conocimiento de la arquitectura con las que se suele relacionar directamente. Estos datos podrían servir para realizar una evaluación más objetiva del área en procesos selectivos tal y como otros autores han propuesto al referirse a otras áreas (Gordillo y otros, 2004).

\subsection{El caso de la producción científica en ar- quitectura en España}

En España, las instituciones y los autores se han referido habitualmente a la medición del rendimiento de la investigación debido a su relevancia en procesos de evaluación académica como la concesión de proyectos de investigación o la promoción del profesorado universitario. Para ello, las agencias evaluadoras utilizan indicadores bibliométricos entre los que destacan la cantidad de publicaciones internacionales de prestigio como muestra de productividad y sus citas como reflejo de la visibilidad y difusión (Jiménez-Contreras y otros, 2011). De hecho, la Comisión Nacional de Evaluación de la Actividad Investigadora (CNEAI) ha venido mostrando una clara preferencia hacia artículos publicados en revistas extranjeras, lo que se refleja en un aumento de publicaciones de este tipo desde 1990 (Jiménez-Contreras y otros, 2003).

La Agencia Nacional de Evaluación de la Calidad y Acreditación (ANECA) se ocupa de realizar la evaluación de acreditaciones para figuras de profesor titular y catedrático de universidad. La evaluación de la producción científica, en general, se refiere a los artículos indexados en Journal Citation Reports (JCR). Desde enero de 2020, para el área de arquitectura, se indica la preferencia de este tipo de publicaciones, aunque se siguen admitiendo artículos en Scopus, pudiendo considerar incluso otras bases de de datos como el Avery Index.

El caso de las publicaciones científicas en arquitectura es ciertamente preocupante partiendo de que, a pesar de que JCR se organiza en 236 categorías, ninguna de ellas se refiere de manera específica a la arquitectura, lo que evidencia su baja presencia a pesar de su importancia en nuestra so- ciedad. Esta situación se refleja en el escenario español, ya que esta área se evalúa en conjunto con ingeniería civil, por lo que no hay datos aislados del objeto de estudio. La propia Fundación Española para la Ciencia y la Tecnología (FECYT) realiza periódicamente análisis globales a partir de la base de datos Scopus del área de ingeniería compuesta por 16 titulaciones, entre las que se encuentra arquitectura, situando al conjunto como la segunda área más productiva en España con un alcance de publicaciones internacionales en torno al $14 \%$ de la producción total del país (FECYT, 2017).

Se ha constatado que un tercio de los investigadores españoles de ingeniería civil y arquitectura que solicitaban proyectos nacionales entre 2000 y 2006 no tenía ningún artículo indexado en JCR (Jiménez-Contreras y otros, 2011), y que la media anual era de 0,8 artículos. Lo que llama la atención es que esta población sólo constaba de 61 personas, frente a otras áreas como Biología con 405, de las cuales 400 eran productivas en términos científicos. Además, el porcentaje de publicación en revistas indexadas en cuartiles altos era bajo, ya que se publicaba mucho en actas de congresos. Estos autores revelaron que perfiles de naturaleza aplicada como estos podrían precisar otros indicadores para evaluar su rendimiento. Dichos resultados, previos al estudio de la FECYT, aportan una visión muy diferente que puede radicar en la base de datos utilizada.

Un estudio más cercano al área de EGA, como es el área de Expresión Gráfica en la Ingeniería evidenció también un nivel de producción científica bajo en revistas indexadas (Rojas-Sola y otros, 2008). Asimismo, se realizó un análisis sobre el profesorado con titulación de arquitectura técnica que registraba el nivel de producción bajo hasta el año 2000, con un incremento en los últimos años (Durán-Álvarez, 2016).

Otra investigación se preocupó por la producción investigadora en arquitectura de ocho universidades públicas españolas (Sendra Salas y otros, 2009), indicando que el número de tesis doctorales de EGA fue un $11.5 \%$ en el período 2000 a 2008 . Además, se realizó una encuesta a 282 de sus doctores en la que sólo 47 indicaron no tener publicaciones, mientras que el $37 \%$ habían publicado en revistas indexadas. Sin embargo, en ningún momento se llegó a diferenciar el tipo de indexación.

Esta visión sesgada de lo general a lo particular evidencia un problema a la hora de diagnosticar y evaluar a los investigadores del área, incluso para la búsqueda de referentes de producción científica en personas que aspiren a desarrollar una carrera académica dentro del área de EGA. De hecho, 
investigaciones previas han construido marcos de referencia (o benchmarks) con el propósito de mejorar la objetividad de la evaluación (Gordillo y otros, 2004; Jiménez-Contreras y otros 2011).

\subsection{Factores relacionados con la producción científica}

Algunos factores que podrían explicar estos resultados son el "entrenamiento" investigador (grado de doctor) y las atribuciones contractuales de investigación del contrato laboral (figuras académicas).

A pesar de que no se cuestiona la importancia de la tesis doctoral en la formación del personal investigador (Sánchez Jiménez y otros, 2017) hay pocos trabajos que vinculen su potencial en la producción investigadora (Andersen y Hammarfel, 2011). De hecho, la cantidad de tesis de una universidad es fidedigno de la capacidad de formación en investigación (Sanz-Casado y otros, 2011; Buela-Casal y otros, 2015). Sin embargo, Durán Álvarez (2016) señaló que el grado de doctor no era por sí mismo una condición suficiente en el incremento de calidad científica de los manuscritos de investigadores de arquitectura técnica, lo que podría estar relacionado con el hecho de que los controles vigentes de las tesis no garantizan, en realidad, la calidad de las mismas.

Como indican Sánchez Jiménez y otros (2017) es difícil generalizar según esta relación entre tesis y producción investigadora, ya que el hecho de que coexistan de disciplinas muy diferentes produce resultados contradictorios. No obstante, han manifestado una correlación directa con la producción de artículos en bases de datos internacionales, con un patrón exponencial en los últimos años.

Con respecto a las figuras académicas, la Ley de Ordenación de Universidades (Ley Orgánica de Universidades, 2001) diferencia entre funcionario y contratado laboral. El segundo incorpora figuras que comparten objetivos de investigación y docencia un doctorado y una acreditación, como es el caso de las figuras de contratado doctor y ayudante doctor.

Pero también se incluyen figuras como la del profesor asociado, cuyo propósito meramente docente es incorporar conocimiento práctico de la realidad profesional en la Universidad con una dedicación a tiempo parcial. Esta figura ha sido muy habitual en titulaciones de ingeniería y arquitectura (Estruch y Silva, 2006), cuya baja dedicación en el caso de las escuelas de arquitectura podría estar influyendo en la producción científica, ya que tanto investigadores de ingeniería como de arquitectura destacan por considerar insuficiente el número de colaboradores en estas tareas (Gómez Ferreri y González Alcaide, 2018).

\subsection{Objetivos}

En un escenario en el que la enseñanza en EGA no parece haber resuelto todavía las solicitudes del Espacio Europeo de Educación Superior (López-Pena y otros, 2016), la diseminación y generación de investigación aplicada es fundamental. Por ello, el objetivo de este trabajo es analizar la producción científica del profesorado de EGA en titulaciones de arquitectura de todas las universidades públicas españolas y examinar la influencia de la formación en investigación o la figura académica.

\section{MÉTODOS Y MATERIALES EMPLEADOS}

El diseño de esta investigación es empírico documental por observación indirecta y bajo un enfoque cuantitativo. Para ello se realizarán análisis descriptivos y de varianza.

\subsection{Procedimiento}

En primer lugar, se realiza una búsqueda de todo el profesorado de EGA de las escuelas de arquitectura de universidades públicas españolas a través de la página web de cada uno de sus departamentos. Los profesores analizados se corresponden con aquellos cuyas webs departamentales aportaban información sobre el área de adscripción del profesor en el momento de la recolección de información. Se generó una base de datos con el nombre y figura académica de cada miembro del área y se realizó la búsqueda de obtención del grado de doctor y su año de defensa en la plataforma Teseo.

En cuanto a la producción científica, se ha seleccionado la base de datos Scopus como la más idónea para generar una visión general del área EGA siguiendo la coherencia expuesta en el apartado anterior. Esta base de datos incluye el índice Scimago Journal \& Country Rank (SJR), que sí contempla arquitectura como categoría temática, incluyendo 144 revistas.

Se buscaron los perfiles de cada profesor en esta base de datos, teniendo en cuenta las diferentes formas que pueden adoptar los nombres españoles en las bases de datos del ISI (Ruiz-Pérez y otros, 2002), de la cual se ha obtenido la producción científica del investigador.

Se realizaron análisis descriptivos de frecuencia y media para representar gráficamente el estado y evolución de la producción científica en EGA. Después, se aplicaron análisis de contingencia a través de gráficos de burbujas para profundizar en cómo las frecuencias de la producción se distribuyen en función del entrenamiento académico y de la figura académica. Finalmente, se aplicaron análisis de varianza para comprobar si las comparativas des- 
criptivas reportaban diferencias significativas (en al menos el 95 por ciento de los casos).

El área de Expresión Gráfica Arquitectónica de las escuelas de arquitectura públicas españolas se compone en 2019 de 292 profesores (ver Tabla I), de los cuales 167 son doctores. La distribución por figura contractual se expone en la Tabla II, en la que destaca que 105 profesores tienen un contrato que exige el grado de doctor, de los cuales 75 han publicado al menos un artículo indexado en SJR.

\subsection{Muestra}

Tabla I. Distribución de la muestra por universidad. Año 2019

\begin{tabular}{|l|c|c|}
\hline Universidad & Acrónimo & $\begin{array}{c}\text { No de } \\
\text { profesores }\end{array}$ \\
\hline $\begin{array}{l}\text { Universidad del País Vasco/ } \\
\text { Euskal Herriko Unibertsitatea }\end{array}$ & EHU & 8 \\
\hline Universidad de Alicante & UA & 9 \\
\hline $\begin{array}{l}\text { Universidad de Alcalá de } \\
\text { Henares }\end{array}$ & UAH & 9 \\
\hline Universidad de Girona & UDG & 6 \\
\hline Universidade da Coruña & UDC & 17 \\
\hline Universidad de Granada & UGR & 15 \\
\hline $\begin{array}{l}\text { Universidad de las Palmas de } \\
\text { Gran Canaria }\end{array}$ & ULPGC & 18 \\
\hline Universidad de Málaga & UMA & 8 \\
\hline $\begin{array}{l}\text { Universidad Politécnica de } \\
\text { Cartagena }\end{array}$ & UPCT & 13 \\
\hline $\begin{array}{l}\text { Universidad Politécnica de } \\
\text { Cataluña }\end{array}$ & UPC & 50 \\
\hline $\begin{array}{l}\text { Universidad Politécnica de } \\
\text { Madrid }\end{array}$ & UPM & 52 \\
\hline $\begin{array}{l}\text { Universidad Politécnica de } \\
\text { Valencia }\end{array}$ & UPV & 14 \\
\hline Universidad Rey Juan Carlos & URJC & 10 \\
\hline Universidad de Sevilla & US & 38 \\
\hline Universidad de Valladolid & UVA & 16 \\
\hline Universidad de Zaragoza & UNIZAR & 9 \\
\hline Universidad Rovira i Virgili & URV & $*$ \\
\hline Total & & 292 \\
\hline
\end{tabular}

* La web del departamento no incluía el área de adscripción de cada profesor en el período de la recolección de la información.

\subsection{Variables}

Las variables categóricas son el entrenamiento investigador (medido a través del grado de doctor) y la figura académica. La variable dependiente es la producción científica, medida a través de la cantidad de artículos de los perfiles de la base de datos Scopus hasta los incluidos en 2019.
Tabla II. Distribución de la muestra por figura académica. Año 2019

\begin{tabular}{|l|c|c|c|}
\hline \multirow{2}{*}{$\begin{array}{l}\text { Figura } \\
\text { académica }\end{array}$} & \multicolumn{3}{|c|}{ Cantidad de profesorado de EGA } \\
\cline { 2 - 4 } & Población & Doctores & $\begin{array}{c}\text { Productivos } \\
\text { en SJR }\end{array}$ \\
\hline $\begin{array}{l}\text { Catedrático de } \\
\text { Universidad }\end{array}$ & 11 & 11 & 11 \\
\hline $\begin{array}{l}\text { Titular de } \\
\text { Universidad }\end{array}$ & 59 & 59 & 39 \\
\hline $\begin{array}{l}\text { Titular de } \\
\text { Escuela } \\
\text { Universitaria }\end{array}$ & 21 & 10 & 5 \\
\hline $\begin{array}{l}\text { Contratado } \\
\text { Doctor }\end{array}$ & 11 & 11 & 15 \\
\hline $\begin{array}{l}\text { Ayudante } \\
\text { Doctor }\end{array}$ & 31 & 6 & 24 \\
\hline Colaborador & 123 & 21 & 26 \\
\hline Asociado & 4 & 0 & 1 \\
\hline $\begin{array}{l}\text { Contratado } \\
\text { predoctoral }\end{array}$ & 292 & 143 & 10 \\
\hline $\begin{array}{l}\text { Profesor } \\
\text { sustituto } \\
\text { interino }\end{array}$ & & & 1 \\
\hline Total & 24 & & \\
\hline
\end{tabular}

La producción científica en Scopus contiene diferentes tipos de publicación que se analizarán para ofrecer una visión global. No obstante, la medida que se utilizará para la mayoría de los análisis será la cantidad de artículos, ya que es precisamente el que la ANECA valora en el apartado investigador de las acreditaciones de cuerpos docentes universitarios (catedrático y titular de universidad). Si bien es cierto que hay capítulos de libro en editoriales de primer cuartil, estas publicaciones normalmente proceden de congresos, norma excluyente de su valoración por la institución evaluadora.

\section{RESULTADOS}

\subsection{Análisis descriptivos de la producción científica del profesorado de EGA en SJR}

La cantidad total de manuscritos indexados en Scopus es de 668: 388 artículos (media por investigador $=1,29$ ) de los cuales 158 (el 40,7\%) pertenecen a EGA Revista de Expresión Gráfica Arquitectónica, 16 capítulos de libro, 149 actas de congreso, 90 artículos de revisión y 25 de otro tipo.

La frecuencia de la producción científica se muestra cronológicamente en la Figura 1 con una tendencia ascendente que podríamos dividir en tres etapas: de 2001 a 2010 , de 2010 a 2016 y de 2017 a 2019. En la primera apenas hay publicaciones indexadas en esta base de datos. En la segunda, 
Figura 1. Distribución cronológicas de las publicaciones en Scopus del profesorado de EGA

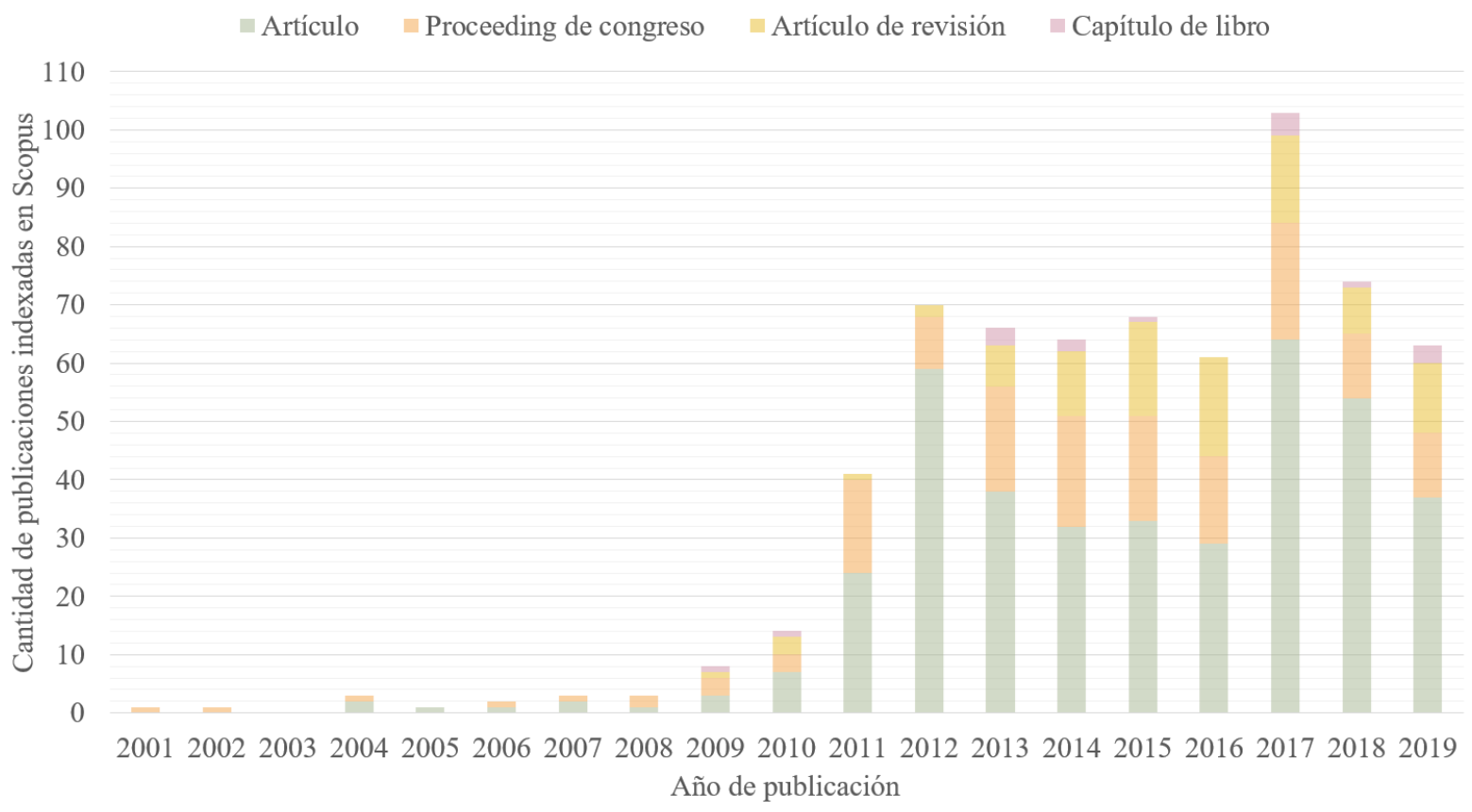

Figura 2. Distribución de profesorado de EGA por producción científica y grado de doctor.

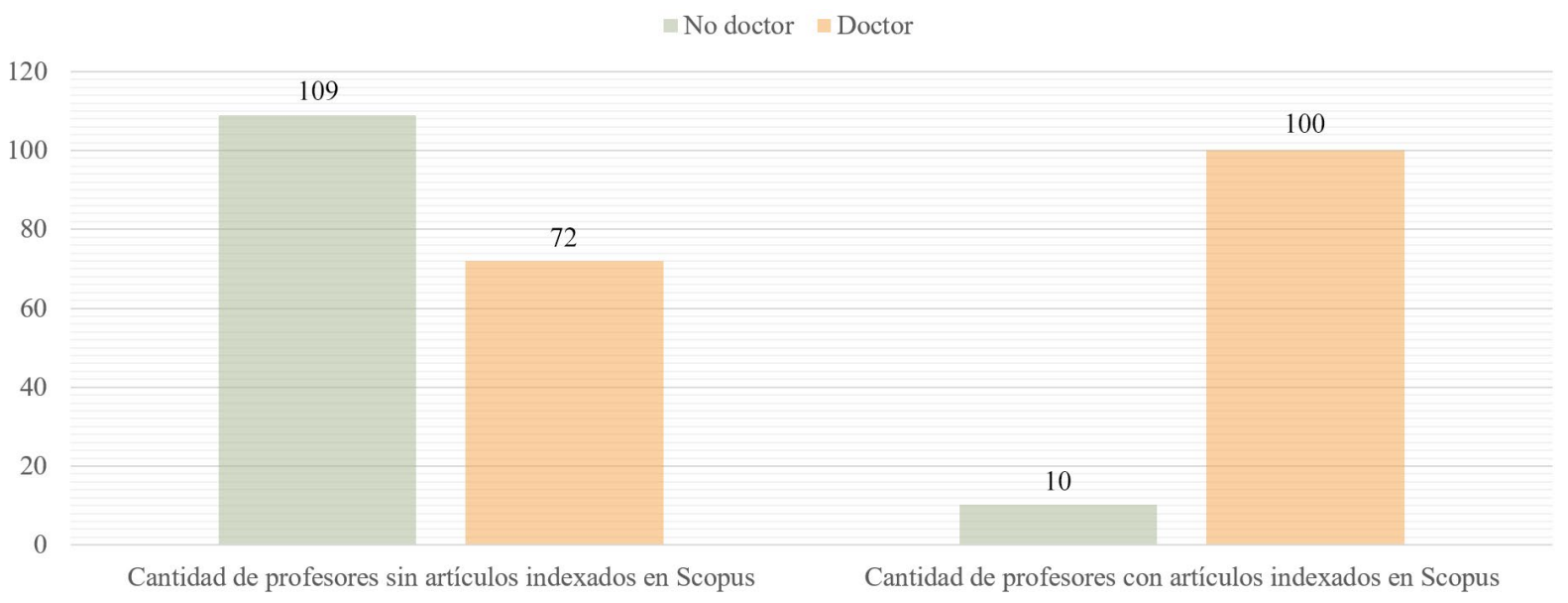

coincidente con la entrada de la Revista EGA en Scopus, se publica una media de 37 artículos por año. Y en la tercera, la cantidad asciende a una media de 52 artículos anuales. Si dividimos esta última cifra entre el número de profesores del área, se obtiene una media de 0,17 artículos por año en la mejor de las tres etapas.

Si comparamos la cantidad de publicaciones dependiendo del entrenamiento investigador a través del grado de doctor, sólo el 9\% (11/120) de profesores sin formación de doctor tienen artículos en revistas registradas en esta base de datos, frente al 58\% (100/172) en el caso de los doctores (ver Figura 2).

En cuanto a los profesores productivos, aquellos sin grado de doctor han publicado entre 1 y 4 artículos, mientras que los doctores han publicado entre 1 y 22 artículos (ver Figura 3).

Debido a la relevancia de la Revista EGA en la producción científica de este conjunto de profesorado, se ha realizado un análisis de contingencia que diferencia aquellos autores que sólo 
Figura 3. Cantidad de artículos publicados agrupados por grado de doctor

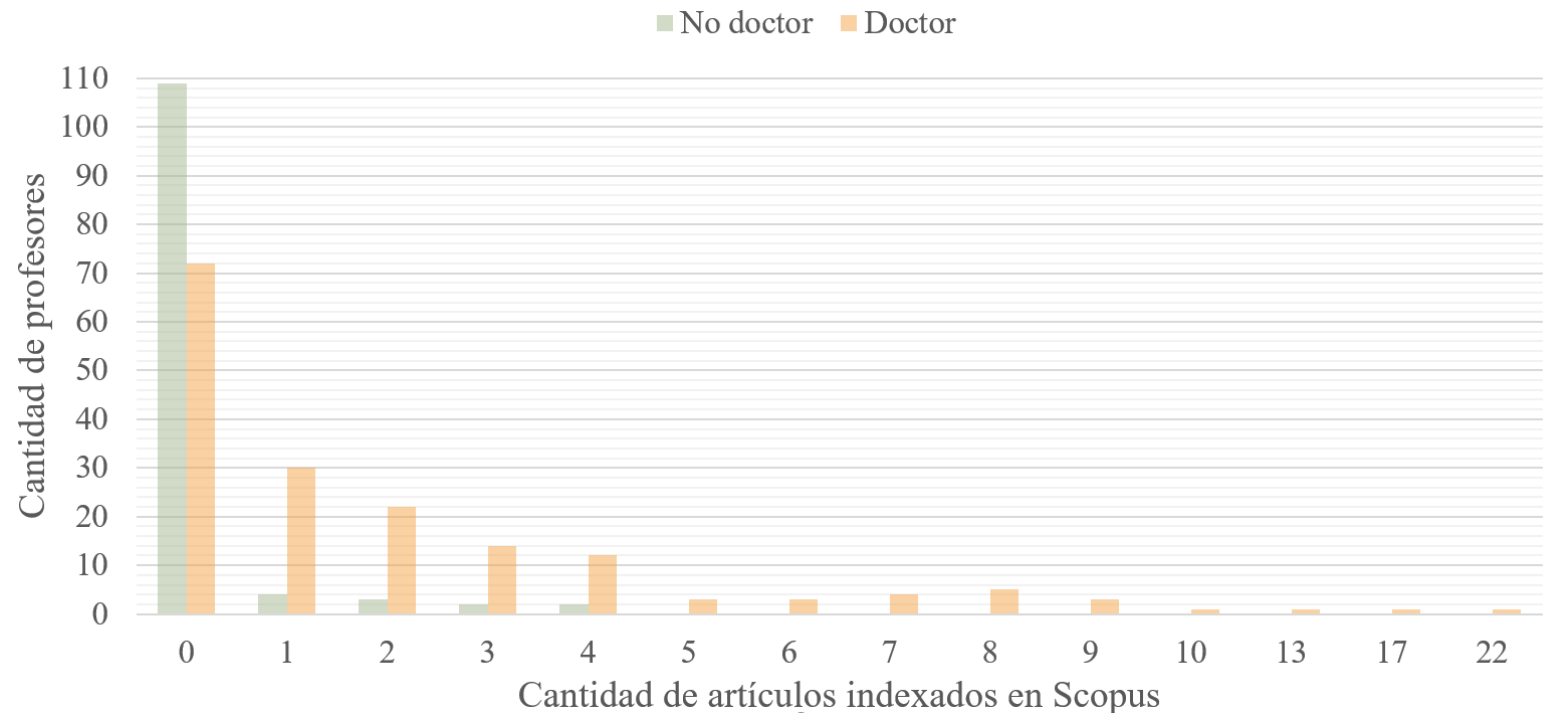

han publicado en la mencionada revista, aquellos que no han publicado en la misma y los que lo han hecho en ambos grupos (ver Tabla III). El resultado muestra los tres grupos de autores, relacionándose con una mayor cantidad de contribuciones científicas aquellos que publican en otras revistas internacionales, sobre todo aquellos que combinan esa trayectoria con la de la Revista EGA.

Tabla III. No de Autores según su producción científica en la revista EGA

\begin{tabular}{|c|c|c|}
\hline N & $\begin{array}{c}\text { Cantidad de } \\
\text { artículos en la } \\
\text { Revista EGA }\end{array}$ & $\begin{array}{c}\text { Cantidad de artículos } \\
\text { en otras revistas } \\
\text { internacionales }\end{array}$ \\
\hline 29 & Entre 1 y 4 & 0 \\
\hline 32 & 0 & $\begin{array}{c}\text { Entre } 1 \text { y } 10 \\
\text { (cuatro casos por encima de 5) }\end{array}$ \\
\hline 44 & Entre 1 y 7 & $\begin{array}{c}\text { Entre } 1 \text { y } 19 \\
\text { (siete casos por encima de 5) }\end{array}$ \\
\hline
\end{tabular}

\subsection{Producción en función de la figura aca- démica}

Si examinamos la producción científica según el rango académico (ver Tabla IV), el valor medio más alto corresponde a los catedráticos de universidad, ayudantes doctores, titulares de universidad y contratados doctores. Mientras que los profesores sustitutos interinos, colaboradores, titulares de escuela universitaria y asociados tienen una producción media menor.
Tabla IV. Valores medios de la producción científica por número de autores y figura académica

\begin{tabular}{|l|c|c|}
\hline Figura académica & n & $\begin{array}{c}\text { Media de } \\
\text { cantidad de } \\
\text { artículos en SJR }\end{array}$ \\
\hline Catedrático de Universidad & 11 & 5,09 \\
\hline Titular de Universidad & 59 & 2,83 \\
\hline $\begin{array}{l}\text { Titular de Escuela } \\
\text { Universitaria }\end{array}$ & 21 & 0,33 \\
\hline Contratado Doctor & 24 & 1.58 \\
\hline Ayudante Doctor & 11 & 3,36 \\
\hline Colaborador & 31 & 0,19 \\
\hline Asociado & 123 & 0,51 \\
\hline Contratado predoctoral & 4 & 0,75 \\
\hline Profesor sustituto interino & 8 & 0,125 \\
\hline
\end{tabular}

Para profundizar en la distribución de estos resultados, se ha calculado la cantidad de profesorado según la correlación de producción científica (eje $\mathrm{x}$ ) y la figura académica (eje y). Esta distribución se realiza en un diagrama de burbujas (ver Figura 4), cuyo radio representa la cantidad de profesorado. Además, se ha diferenciado con colores la exigencia de la titulación de doctor para cada figura académica. Los resultados obtenidos se diferencian en tres rangos de publicación de artículos.

- De 0 a 22 publicaciones (media=3,18): catedrático de universidad y titular de universidad. A pesar de un rango mayor, hay una gran cantidad de profesorado acumulado en los valores más 
Figura 4. Diagrama de burbujas de la cantidad de profesorado de EGA según la relación de producción científica y figura contractual.

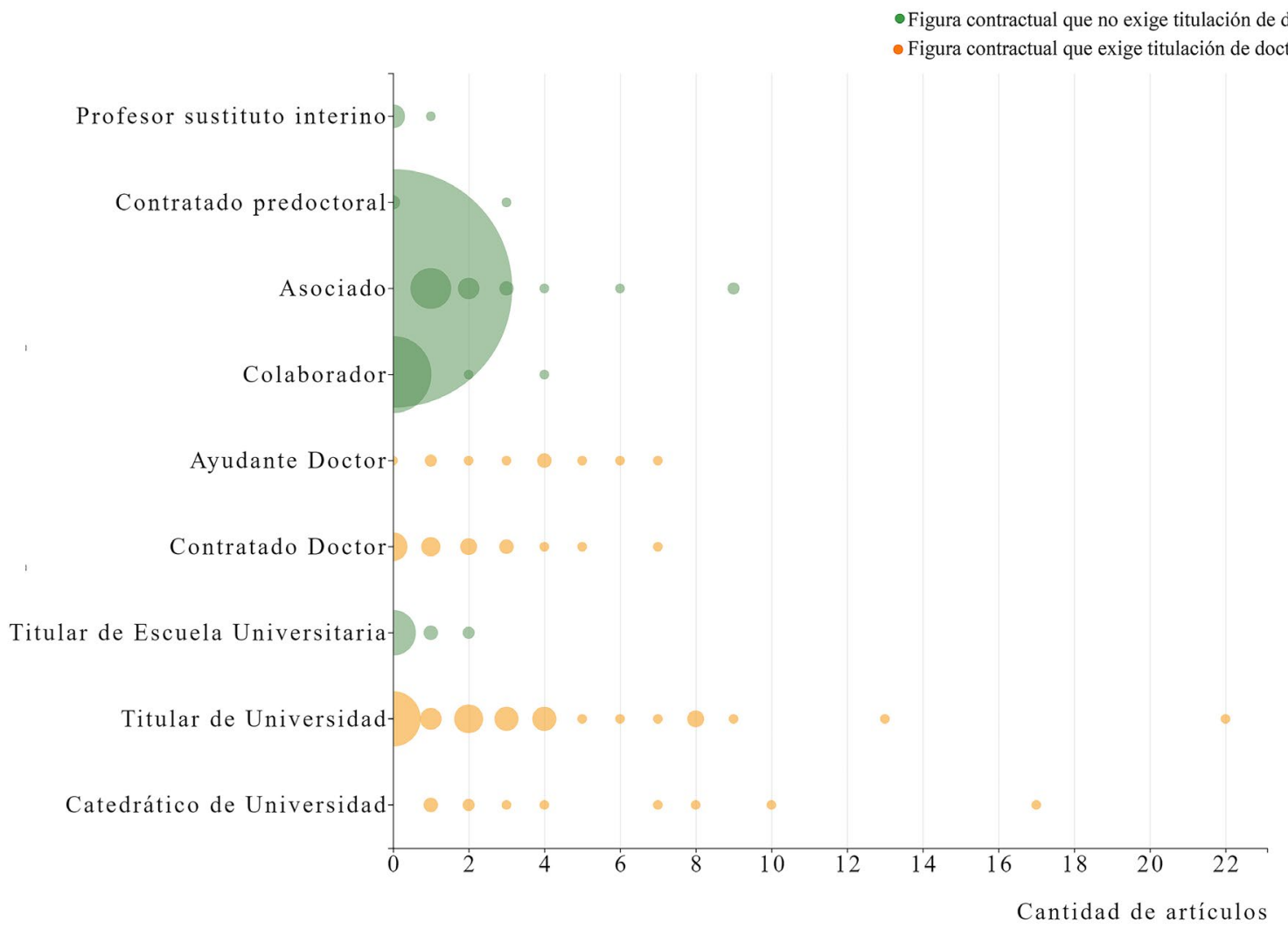

bajos de publicación ( 36 titulares de universidad con entre 0 y 2 artículos).

- De 0 a 7 publicaciones (media = 2,14): profesorado contratado doctor y ayudante doctor.

- De 0 a 4 publicaciones (media $=0,42$ ): profesorado sustituto interino, asociado, colaborador, titular de escuela universitaria y contratado predoctoral. Si bien es cierto que hay dos profesores asociados con más publicaciones, son valores atípicos (outliers) frente a los otros $96 \mathrm{sin}$ ninguna publicación.

\subsection{Producción en función del grado de doc- tor y año de defensa de tesis doctoral}

Se ha calculado el valor medio de producción científica dependiendo de si el profesor tiene el grado de doctor o no (ver Tabla $\mathrm{V}$ ), resultando que

Tabla V. Valores medios de producción científica según la formación investigadora

\begin{tabular}{|l|c|c|}
\hline & n & $\begin{array}{c}\text { Cantidad media de } \\
\text { artículos en SJR }\end{array}$ \\
\hline Profesorado doctor & 167 & 2,12 \\
\hline Profesorado no doctor & 125 & 0,19 \\
\hline
\end{tabular}

aquellos con tal formación investigadora producen diez veces más. No obstante, es preciso tener en cuenta que esta media de artículos publicados en SJR es de 2,12.

A continuación, se ha centrado la atención en el profesorado con grado de doctor. Para ello se ha analizado el número de profesores en función de la relación entre el número de artículos (eje y) y el año de defensa de la tesis doctoral (eje $\mathrm{x}$ ) (ver Figura 5). Los resultados evidencian una tendencia descendente debido a una gran cantidad de profesores que se han doctorado en los últimos años sin producción científica (seis en 2015, diez en 2016 y seis en 2017). En otras palabras, aquellos docentes que se doctoraron en años anteriores al 2000 han producido más artículos indexados que aquellos que defendieron su tesis en los últimos veinte años.

Asimismo, los investigadores con menos publicaciones en los últimos años tienen una figura académica sin exigencia de grado de doctor y en su mayoría de relación contractual a tiempo parcial, mientras que la normalidad de quienes se sitúan por encima de la línea de tendencia de 
Figura 5. Diagrama de burbujas de la cantidad de profesorado de EGA según la relación entre el año de defensa de su tesis doctoral y la producción científica.

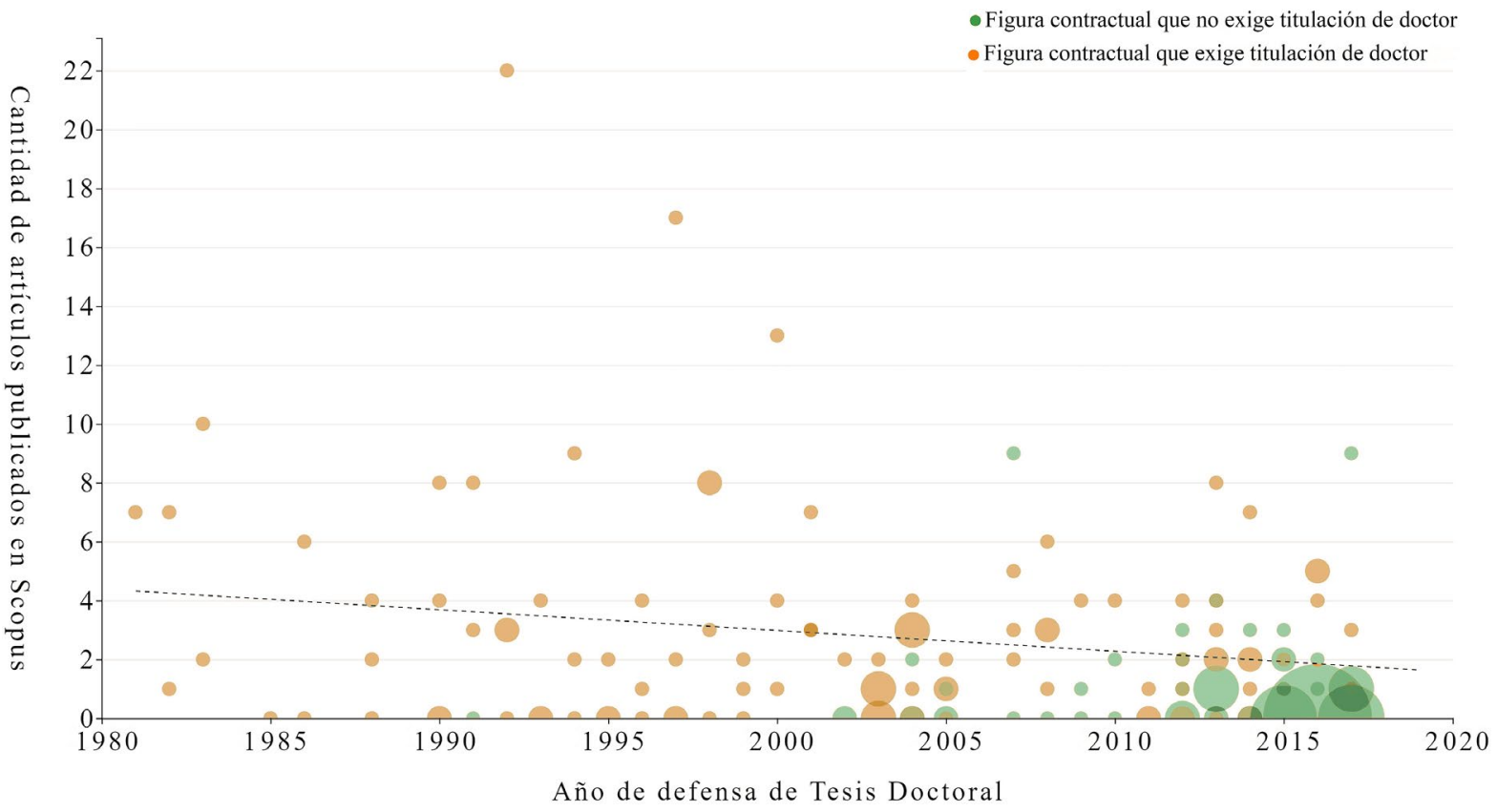

producción científica en la última década se corresponden con una figura académica que exige el grado de doctor.

Estos resultados evidencian una existencia de dualidad en el área referente a los perfiles docentes. Por una parte, se encontraría la figura del docente investigador con un nivel similar de publicaciones indexadas al del profesorado que se doctoró antes del 2000, pero con una trayectoria más reducida en el tiempo, por lo que a pesar de mantenerse en cifras absolutas la producción, realmente se estaría incrementando. Por otra parte, se encontraría el perfil vinculado a la práctica profesional y más alejado de la función investigadora siendo estos un número mucho mayor que genera un descenso en la línea de producción y como consecuencia la trayectoria de la línea de tendencia es descendente.

\subsection{Análisis de varianza}

El análisis de varianza permite evaluar si las diferencias obtenidas en pruebas descriptivas suceden de manera estadísticamente significativa en relación a las categorías formación investigadora y rango académico. En primer lugar, es necesario calcular si la producción científica se distribuye siguiendo una distribución normal, la cual no ha mostrado ser normal según el test de Kolmogorov-Smirnov $(p<0,001)$, por lo que se realizarán test no paramétricos.
En relación a la formación investigadora, se ha calculado la prueba de Mann-Whitney (Tabla VI), obteniendo que la producción de los profesores doctores es superior de manera estadísticamente significativa $(p<0,001)$.

Tabla VI. Prueba M-W a profesores doctores o no doctores

\begin{tabular}{|l|c|c|c|c|c|}
\hline & $\mathbf{n}$ & $\begin{array}{c}\text { U de } \\
\text { Mann- } \\
\text { Whitney }\end{array}$ & $\begin{array}{c}\text { Wilcoxon } \\
\text { W }\end{array}$ & $\mathbf{Z}$ & $\mathbf{p}$ \\
\hline $\begin{array}{l}\text { Producción } \\
\text { científica }\end{array}$ & 292 & 5001,00 & 12876,00 & $-8,739$ & $<0,001$ \\
\hline
\end{tabular}

Por su parte, se ha aplicado el test de Kruskal-WaIlis para el caso de la figura académica (Tabla VII). Los resultados indican que, para el profesorado de EGA, la producción científica varía con significancia estadística con dependencia de la figura académica del profesor $(p<0,001)$.

Tabla VII. Resultados de prueba Kruskal-Wallis según figura academica.

\begin{tabular}{|c|c|c|c|c|}
\hline & $\mathbf{n}$ & $\begin{array}{c}\text { H de Kruskal- } \\
\text { Wallis }\end{array}$ & gl & p \\
\hline Producción científica & 292 & 99,105 & 8 & $<0,001$ \\
\hline
\end{tabular}


Tabla VIII. Resultados de grupos de pares de la prueba Kruskal-Wallis que evidenciaron diferencias estadísticamente significativas (FCx-FCy).

\begin{tabular}{|l|l|c|c|}
\hline \multicolumn{1}{|c|}{ FCx } & \multicolumn{1}{|c|}{ FCy } & Diferencia de medias (FCx-FCy) & p \\
\hline \multirow{5}{*}{ Catedrático de Universidad } & Titular de Escuela Universitaria & 4,785 & $<0,001$ \\
\cline { 2 - 4 } & Contratado Doctor & 3,508 & 0,001 \\
\cline { 2 - 4 } & Colaborador & 4,897 & $<0,001$ \\
\cline { 2 - 4 } & Asociado & 4,579 & $<0,001$ \\
\cline { 2 - 4 } & Contratado predoctoral & 4,341 & 0,038 \\
\cline { 2 - 4 } & Sustituto Interino & 4,966 & $<0,001$ \\
\hline \multirow{4}{*}{ Titular de Universidad } & Titular de Escuela Universitaria & 2,497 & $<0,001$ \\
\cline { 2 - 4 } & Colaborador & 2,637 & $<0,001$ \\
\cline { 2 - 4 } & Asociado & 2,318 & $<0,001$ \\
\hline \multirow{4}{*}{ Ayudante Doctor } & Titular de Escuela Universitaria & 3,030 & 0,014 \\
\cline { 2 - 4 } & Colaborador & 3,170 & 0,004 \\
\cline { 2 - 4 } & Asociado & 2,851 & 0,003 \\
\hline
\end{tabular}

Los resultados previos no diferencian entre qué figuras académicas existe esa diferencia significativa en la producción científica. Para conocer esta información, se ha aplicado la prueba de Kruskal-Wallis a través de la selección de cada par de grupos (por ejemplo, la figura de catedrático con respecto a la de titular de universidad, la de catedrático con respecto a la de titular de escuela universitaria, etc.). La Tabla VIII proporciona un resumen de los resultados de aquellas agrupaciones que sí han mostrado diferencias significativas.

Los resultados indican que la figura de catedrático de universidad produce de manera clara más publicaciones científicas que aquellos profesores titulares de escuela universitaria, contratados doctores, colaboradores, asociados, contratados predoctorales y sustitutos interinos. En el caso de los profesores titulares de universidad de EGA producen más artículos en SJR que aquellos profesores titulares de escuela universitaria, colaboradores y asociados. Asimismo, los ayudantes doctores también publican más artículos que los titulares de escuela universitaria, colaboradores y asociados de manera estadísticamente significativa.

\section{DISCUSIÓN}

La Expresión Gráfica Arquitectónica es un área fundamental de la arquitectura que, como cualquier otro ámbito de conocimiento, precisa una continua observación y evolución de cara a responder mejor a todo tipo de necesidades: profesionales, sociales, educativas, etc. Las agencias evaluadoras miden el grado de adecuación de esta labor investigadora a través de la cantidad de artículos científicos indexados en bases de datos como JCR o SJR.
A pesar del carácter particularmente artístico del área EGA, la producción científica de su profesorado se ha medido normalmente en conjunto con la mayoría de las titulaciones de ingeniería. Por lo que es normal obtener resultados muy sesgados y contradictorios que no establecen los baremos más ajustados en la evaluación de los perfiles de su profesorado.

Como se ha indicado en la introducción, la propia FECYT ha incluido la producción científica de arquitectura integrada con otras 15 titulaciones de ingeniería, como el segundo grupo de áreas con mayor producción científica en España en índices SJR (FECYT, 2017). Este resultado aparentemente prometedor se contrapone a un estudio previo, pero centrado sólo en ingeniería civil y arquitectura, que indicaba que aquellos profesores más competitivos en términos de solicitud de proyectos de I+D tenían una media de artículos en JCR de 0,8 (Jiménez-Contreras y otros, 2011), apoyado por resultados de baja producción científica en el área cercana de Expresión Gráfica en la Ingeniería (Rojas Solas y otros, 2008), o en el conjunto de profesores de arquitectura técnica con un aumento en los últimos años (Durán-Álvarez, 2016). Este análisis permite comprender el salto existente con los años de diferencia de la literatura previa, comprobando un crecimiento desde 2011 de la producción científica, coincidente con la entrada de la Revista EGA en SJR. No obstante, los resultados medios de publicación de artículos en esta base de datos siguen siendo bajos.

Investigaciones previas indican una correlación entre la producción científica y la formación investigadora (Sánchez Jiménez y otros, 2017), lo cual 
puede parecer un indicio ya que, en el período de 2000 a 2008, sólo el 11 por ciento de las tesis doctorales en arquitectura en España estaban adscritas al área de EGA (Sendra Salas y otros, 2009). En este caso, se ha constatado que más de la mitad del profesorado no es doctor, así como que esta formación sí influye de manera estadísticamente significativa en la publicación de artículos científicos en estas bases de datos, a pesar de haber sido demostrado en resultados previos en el área de arquitectura técnica que la existencia de muchos profesores doctores no es condición suficiente para dicha correlación (Durán Álvarez, 2016). Las conclusiones de la presente investigación refuerzan la argumentación anterior ya que el $42 \%$ de los doctores no han participado en ninguna publicación y un $29 \%$ aportan entre una y dos publicaciones. Los motivos que subyacen tras estos porcentajes, lejos de poder ser extraídos de los datos, podrían estar relacionados con el vínculo de la docencia en arquitectura y su labor profesional, la falta de tradición investigadora y el carácter no obligatorio de la producción científica en la profesión docente universitaria.

Además, la internacionalización del investigador, medida por la diferencia de artículos publicados en revistas internacionales diferentes a la revista tradicional del área, también se correlaciona con una mayor producción en general, sobre todo cuando se produce una combinación con la mencionada revista.

Entre aquellos profesores con formación investigadora, se ha evidenciado una correlación entre el año de defensa de la tesis doctoral y la cantidad de artículos publicados, siendo mayor cuanto más tiempo ha transcurrido. Este dato puede parecer muy coherente, pero hay que compararlo con el análisis cronológico de producción científica, ya que, a pesar de haber defendido una tesis doctoral en el año 1980, no han comenzado a publicar en esta base de datos hasta prácticamente el año 2011. Por otra parte, esta tendencia no parece tan clara ya que se ve muy influida a la baja entre los años 2015 y 2017 en los que se han defendido muchas tesis doctorales cuyos autores, con relación contractual parcial y vinculación con su labor profesional de arquitecto, no cuentan con ninguna publicación indexada en SJR. Asimismo, en este período temporal se observa que, en general, a profesores más productivos corresponden figuras académicas de mayor nivel. Estos datos evidencian una vía de acceso a la docencia en las titulaciones de arquitectura a partir de la figura académica de profesor asociado y una ausencia de perfiles académicos de contratados predoctorales.

Es necesario incidir en que esta lectura responde a las normas evaluadoras que actualmente norma- liza la ANECA para evaluar el perfil investigador, pero que la producción real de los profesores será muy superior en otro tipo de publicación o indexación que actualmente no se valoran en este perfil.

En cualquier caso, los resultados medios de producción científica, que son bajos, descenderían drásticamente si no se tuviesen en cuenta aquellos profesores con mayor cantidad de artículos publicados, existiendo valores atípicos de entre 10 y 22 artículos indexados en SJR.

Asimismo, la figura académica también ha demostrado ser un factor clave. Este hecho, acompañado de la gran cantidad de profesorado contratado sin exigencia del grado de doctor (que representa casi dos tercios del profesorado de EGA) o de dedicación parcial al mundo académico, puede explicar el bajo rendimiento medio en esta área del conocimiento. No obstante, es necesario tener en cuenta que las titulaciones que habilitan para el ejercicio de una profesión como la arquitectura precisa una conexión fundamental con el mundo profesional, siendo fundamental encontrar el equilibrio entre el desarrollo científico y el conocimiento laboral.

\section{CONCLUSIONES}

Esta investigación ha evidenciado que la formación investigadora se relaciona directamente con la producción científica de artículos indexados en SJR del profesorado de Expresión Gráfica Arquitectónica de las escuelas técnicas superiores de arquitectura públicas de España.

Además, los resultados han permitido determinar qué figuras académicas tienen mejor producción científica, lo que puede ser útil como marcos de referencia más objetivos en procesos de evaluación de los perfiles investigadores de esta área del conocimiento. Estos hallazgos no generan un conflicto con las acreditaciones proporcionadas por la actual agencia de acreditación ya que es posible que algunos profesores ya posean una acreditación superior, pero todavía no hayan podido concursar a la figura académica correspondiente. Además de haber conseguido tal acreditación en convocatorias que hayan exigido diferentes criterios de evaluación para cada figura.

\section{REFERENCIAS}

Alonso Rodríguez, M. Á.; Aliberti, L. (2019). Sobre la fotogrametría automatizada aplicada al estudio de bóvedas. EGA. Revista de Expresión Gráfica Arquitectónica, 24(35), 152-159, https://doi.org/10.4995/ega.2019.11495

Andersen, J. P.; Hammarfelt, B. (2011). Price revisited: on the growth of dissertations in eight research fields. Scientometrics, 88(2), 371-383. https://doi. org/10.1007/s11192-011-0408-8 
Amado Lorenzo, A.; Fraga López, F. (2015). The digital draughtsman. Freehand drawing on digital tablets. EGA. Revista de Expresión Gráfica Arquitectónica 2015, (25), 108-119, https://doi.org/10.4995/ ega.2015.3330

Ampliato Briones, A.L. (2012). Apuntes sobre el origen de la línea. EGA. Revista de Expresión Gráfica Arquitectónica, 17(19), 60-67, https://doi.org/10.4995/ ega.2012.1358

Buela-Casal, G.; Quevedo-Blasco, R.; Guillén-Riquelme, A. (2015). Ranking 2013 de investigación de las universidades públicas españolas. Psicothema, 27(4), 317-326.

Carazo Lefort, E. (2018). La maqueta como realidad y como representación. Breve recorrido por la maqueta de arquitectura en los 25 años de EGA. EGA. Revista de Expresión Gráfica Arquitectónica, 23(34), 158-171, https://doi.org/10.4995/ega.2018.10849

Chías, P.; Abad, T. T. (2018). The Botica or Apothecary in the Monastery of San Lorenzo el Real de El Escorial (Madrid, Spain): Written Sources, Historic Drawings, and New Surveys Applied to Architectural Analysis. Buildings, 8(1), 4, https://doi.org/10.3390/buildings8010004

Durán-Álvarez, J.M. (2016). Análisis de la producción científica de los arquitectos técnicos docentes de la universidad española. Revista General de Información y Documentación, 26(1), 165-190, https://doi. org/10.5209/rev_RGID.2016.v26.n1.53039

Estruch, V.; Silva, J. (2006). Aprendizaje basado en proyectos en la carrera de Ingeniería Informática. Actas de las XII Jornadas de la Enseñanza Universitaria de la Informática (JENUI, 2006), 339-346. Bilbao, España.

Ferrer-Pérez-Blanco, I.; Gámiz-Gordo, A.; Reinoso-Gordo, J.F. (2019). New Drawings of the Alhambra: Deformations of Muqarnas in the Pendentives of the Sala de la Barca. Sustainability, 11(2), 316, 10.3390/ su11020316

Fonseca, D.; Martí, N.; Redondo, E.; Navarro, I. ; Sánchez, A. (2014). Relationship between student profile, tool use, participation, and academic performance with the use of Augmented Reality technology for visualized architecture models. Computers in Human Behavior, 31, 434-445, https://doi.org/10.1016/j.chb.2013.03.006

Franco Taboada, J.A. (2015). De la teoría de los colores de Goethe a la interacción del color de Albers. EGA. Revista de Expresión Gráfica Arquitectónica, 20(25), 48-55, https://doi.org/10.4995/ega.2015.3703

Franco Taboada, J.A. (2011). Sobre perspectiva, fotografía e infografía. Apuntes para una fenomenología de la representación. EGA. Revista de expresión Gráfica Arquitectónica, 16(17), 54-64, https://doi.org/10.4995/ ega.2011.883

Franco Taboada, J.A.; Goy Diz, A.E. (2018). Renaissance Drawings of the 16th Century in the City of Lugo: Three Fountains from Gonzalo de la Bárcena's Workshop. Buildings, 8(3), 44, https://doi.org/10.3390/ buildings 8030044

Fundación Española para la Ciencia y la Tecnología (2017). Indicadores bibliométricos de la actividad científica española 2005-2014. Madrid.

Gámiz-Gordo, A.; Ferrer Pérez Blanco, I. (2019). Una gramática de mocárabes: Dibujos de la Alhambra de
Jones y Goury (1834-1845). VLC Arquitectura, 6 (2), 57-87, https://doi.org/10.4995/vlc.2019.10947

Gómez-Ferri, J.; González-Alcaide, G. (2018). Patrones y estrategias en la colaboración científica: la percepción de los investigadores. Revista Española de Documentación Científica, 41(1), 199, https://doi.org/10.3989/ redc. 2018.1 .1458

Gordillo, V.; González, J.; Muñiz, J. (2004). La evaluación de Proyectos de Investigación por la Agencia Nacional de Evaluación y Prospectiva. Psicothema, 16 (3), 343349.

Jiménez-Contreras, E.; de Moya Anegón, F.; LópezCózar, E. D. (2003). The evolution of research activity in Spain: The impact of the National Commission for the Evaluation of Research Activity (CNEAI). Research Policy, 32(1), 123-142.

Jiménez-Contreras, E.; Robinson-García, N.; Cabezas-Clavijo, A. (2011). Productividad e impacto de los investigadores españoles: umbrales de referencia por áreas científicas. Revista Española de Documentación Científica, 34, 4, 505-526, https://doi.org/10.3989/ redc. 2011.4 .828

Jordan-Palomar, I.; Tzortzopoulos, P.; García-Valldecabres, J.; Pellicer, E. (2018). Protocol to manage heritage-building interventions using heritage building information modelling (HBIM). Sustainability, 10(4), 908, https://doi.org/10.3390/su10040908

Ley Orgánica de Universidades. BOE no 307 del 24 de diciembre de 2001.

Llopis Verdú, J. (2018a). El paradigma fotográfico del dibujo arquitectónico digital. Arte Individuo y Sociedad, 30(3), 557-573, https://doi.org/10.5209/ARIS.58128

Llopis Verdú, J. (2018b) Entre representación y simulación. Un cambio de paradigma en el dibujo arquitectónico contemporáneo. EGA. Revista de Expresión Gráfica Arquitectónica, 23(34), 180-193, https://doi. org/10.4995/ega.2018.10860

Llopis, J.; Torres, A.; Serra, J.; García, Á. (2015). The preservation of the chromatic image of historical cities as a cultural value. The old city of Valencia (Spain). Journal of Cultural Heritage, 16(5), 611-622, https:// doi.org/10.1016/j.culher.2015.01.005

López-Pena, V.; López-Chao, V.; López-Chao, A. (2016). Analysis of teaching methods in graphic design in the Galician University System (GUS) in Spain. Anthropologist, 25, 214-219, https://doi.org/10.1080/0972007 3.2016.11892109

Marcos Alba, C.L. ; Allepuz Pedreño, Á.; Gutiérrez, J.; Jeremías, P. (2017). Dibujo arquitectónico, intención, percepción y Gestalt. Transparencias, estratificación y polifonía gráfica. EGA. Revista de Expresión Gráfica Arquitectónica, 22(31), 66-79, https://doi.org/10.4995/ ega.2017.7202

Natapov, A.; Kuliga, S.; Dalton, R.C.; Hölscher, C. (2020). Linking building-circulation typology and wayfinding: design, spatial analysis, and anticipated wayfinding difficulty of circulation types. Architectural Science Review, 63(1), 34-46, https://doi.org/10.1080/0003862 8.2019.1675041

Ortega Vidal, J.; Martínez Díaz, Á.; Muñoz de Pablo, M. J. (2012). La representación de la ciudad, entre la planta y el volumen: el plano del Prado y la maqueta 
de la Gran Vía de Madrid. EGA. Revista de Expresión Gráfica Arquitectónica, 17(20), 256-266, https://doi. org/10.4995/ega.2012.1447

Osca-Lluch, J.; González-Sala, F.; Habra-Osca, J.; Tortosa, F.; Peñaranda-Ortega, M. (2019). Scientific communication or a qualification for an academic career? What use is publishing papers in psychology journals? Anales de Psicología, 35(1), 166-174, https://doi. org/10.6018/analesps.35.1.329211

Parra Bañón, J.J. (2008). Dos fragmentos de OTAISA en la Universidad Laboral de Sevilla: cinco gimnasios y un taller en agonía. RA: Revista de Arquitectura, 10, 69-84.

Pinto, F. (2013). Aportaciones al análisis constructivo de fábricas antiguas: La capilla de La Antigua de San Miguel en Morón (1538). Informes de la Construcción, 65(530), 163-174, https://doi.org/10.3989/ic.12.048

Redondo Domínguez, E.; Sánchez Riera, A.; Moya Sala, J. (2012). La ciudad como aula digital: enseñando urbanismo y arquitectura mediante Mobile Learning y la realidad aumentada: un estudio de viabilidad y de caso. Ace: Architecture, City and Environment, 7(19), 27-54, https://doi.org/10.5821/ace.v7i19.2560

Reinoso-Gordo, J.F.; Rodríguez-Moreno, C.; GómezBlanco, A.J.; León-Robles, C. (2018). Cultural heritage conservation and sustainability based on surveying and modeling: The case of the 14th century building Corral del Carbón (Granada, Spain). Sustainability, 10(5), 1370, https://doi.org/10.3390/su10051370

Rojas-Sola, J.I.; Navarrete-Cortés, J.; Fernández-López, J.A.; Chaichio-Moreno, J.A. (2008). Producción científica del área de expresión gráfica en la ingeniería en las universidades españolas: una aproximación a la base de datos ISI. Revista Española de Documentación Científica, 31 (2), 190-204, https://doi.org/10.3989/ redc. 2008.v31.i2.424

Ruiz-Pérez, R.; Delgado-López-Cózar, E.; Jiménez-Contreras, E. (2002). Spanish name variations in national and international biomedical databases: implications for information retrieval and bibliometric studies. Journal of the Medical Library Association, vol. 90 (4), 411430.

Sánchez Jiménez, R.; Blázquez Ochando, M.; Montesi, M.; Botezan, I. (2017). La producción de tesis doctorales en España (1995-2014): evolución, disciplinas, principales actores y comparación con la producción científica en WoS y Scopus. Revista Española de Documentación Científica, 40(4), 188, http://dx.doi.org/10.3989/ redc.2017.4.1409

Sanz-Casado, E.; De Filippo, D.; García Zorita, C.; Efraín-García, P. (2011). Observatorio IUNE: una nueva herramienta para el seguimiento de la actividad investigadora del sistema universitario español. Bordon. Revista de Pedagogía, 63(2), 101-116.

Sendra Salas, J. J.; Navarro Casas, J.; Robador González, M. D.; Muñoz García, A. (2009). Arquitecto versus Doctor Arquitecto: las escuelas de arquitectura y el siglo XXI. $3^{a}$ Jornadas sobre Investigación en Arquitectura y Urbanismo, 1-19.

Soler-Estrela, A. (2018). Cultural Landscape Assessment: The Rural Architectural Heritage (13th-17th Centuries) in Mediterranean Valleys of Marina Alta, Spain. Buildings, 8(10), 140, https://doi.org/10.3390/buildings 8100140 